\title{
CÁNCER DE PRÓSTATA Y CALIDAD DE VIDA: ANÁLISIS DEL CAMBIO EN LA RESPUESTA A PARTIR DE UNA TRIANGULACIÓN ENTRE MÉTODOS
}

\author{
PROSTATE CANCER AND QUALITY OF LIFE: ANALYSIS OF THE RESPONSE SHIFT \\ FROM A TRIANGULATION BETWEEN METHODS
}

Bernat-Carles Serdà y Arantza del Valle

Departamento de Enfermería, Universidad de Girona

Resumen

Introducción. El objetivo de este trabajo es describir el cambio en la Calidad de Vida $(\mathrm{CdV})$ en un grupo de hombres con cáncer de próstata. Los mecanismos implicados en el cambio en la respuesta de la $\mathrm{CdV}$ corresponden al de reconceptualización, repriorización y recalibración.

Material y método. La propuesta metodológica se basa en la triangulación secuencial entre métodos, en que para el estudio de una misma unidad empírica se combinan dos estrategias de investigación, una cuantitativa y otra cualitativa.

Resultados. Se identifica una disminución significativa de la $\mathrm{CdV}$ entre el periodo pretest y postest. A partir del mecanismo de recalibración el thentest confirma que la CdV en el momento inicial de la enfermedad es baja.

Conclusiones. La CdV del hombre con cáncer de próstata es baja en todos los periodos evaluados. El afrontamiento a la enfermedad difiere entre el subgrupo de hombres jóvenes y el subgrupo de hombres mayores.

Palabras clave: Calidad de Vida, cáncer de próstata, cambio en la respuesta.
Abstract

Introduction. The aim of this paper is to describe the changes in the quality of life $(\mathrm{QoL})$ in a group of men with prostate cancer. The three mechanisms involved in the response shift correspond to the reconceptualization, reprioritization and recalibration. Material and methods. Proposed methodology is based on sequential triangulation between methods. To study the same empirical unit it combines two strategies of research: quantitative and qualitative.

Results. We identified a significant decrease in QoL between the pretest and posttest period. The recalibration mechanism with the Thentest confirms that the QoL is low in all the periods evaluated.

Conclusions. The results confirm that QoL in prostate cancer is low in all the periods evaluated. Coping with the disease differs between the subgroup of young men and the subgroup of older men.

Key words: Quality of life, prostate cancer, response shift.

\section{Correspondencia:}

Bernat-Carles Serdà Ferrer.

Universidad de Girona.

Departamento de Enfermería. Emili Grahit, 77. 17071.

Girona.

E-mail: bernat.serda@udg.edu 


\section{INTRODUCCIÓN}

Desde los años 90 en España la incidencia del cáncer de próstata ha aumentado exponencialmente. Este efecto se debe a la utilización del PSA (antígeno prostático específico) como diagnóstico precoz en hombres asintomáticos ${ }^{(1)}$. Los factores determinantes de la disminución de la mortalidad corresponden al tratamiento temprano y la mejora en la eficacia de los tratamientos. La supervivencia a los 5 años de enfermedad es del $85 \%{ }^{(2)}$. Por otro lado, para prevenir una muerte de cáncer de próstata en Europa se deben diagnosticar 48 casos. Estos datos confirman el sobrediagnóstico y el sobretratamiento en el cáncer de próstata de bajo riesgo ${ }^{(3)}$. El subgrupo de hombres más perjudicado por el sobretratamiento es el de mayor edad (+85 años) $)^{(2)}$. Por otro lado los efectos secundarios más habituales relativos al tratamiento del cáncer de próstata corresponden a: la disfunción e impotencia sexual, la incontinencia urinaria y fecal, la fatiga, los problemas psicológicos incluyendo el malestar emocional y la distimia ${ }^{(4-8)}$. En este marco el concepto Calidad de Vida $(\mathrm{CdV})$ pasa a ser un objetivo prioritario en la atención sociosanitaria.

La CdV corresponde a un concepto multidimensional, dinámico y de paradigma modular ${ }^{(9)}$. La CdV la aporta el participante y está influenciada por los mecanismos psicológicos relativos a la capacidad de adaptación a la enfermedad ${ }^{(10)}$. En este proceso influyen los factores personales, los factores de afrontamiento y los factores relativos a la red social ${ }^{(11)}$.

El carácter dinámico, temporal y cambiante de la $\mathrm{CdV}$ produce el efecto de cambio de significado y variación en la valoración del concepto. A la magnitud de este cambio se le denomina: cambio en la respuesta en CdV. Su estudio es fundamental para su interpretación en investigación clínica $^{(12)}$.

Sprangers y Schwartz (1999), identifican que el cambio en la respuesta de la $\mathrm{CdV}$ se produce a partir de tres mecanismos básicos interactivos: el mecanismo de reconceptualización, el mecanismo de repriorización y, el mecanismo de recalibración ${ }^{(13)}$. El mecanismo de reconceptualización corresponde a la redefinición del concepto $\mathrm{CdV}$; El de repriorización, a la variación en la importancia atribuida a las dimensiones que integran el concepto $\mathrm{CdV}$; finalmente, el mecanismo de recalibración, se atribuye al cambio del valor interno de las respuestas del cuestionario. La mejor forma para evaluar el cambio en el valor interno de respuesta es a partir del método thentest. El thentest corresponde al análisis retrospectivo del diseño pretest. Kvam (2010) recomienda que el thentest se realice temporalmente próximo al postest, asumiendo que los participantes valoran las preguntas a partir del mismo estándar interno ${ }^{(14)}$. Por otra parte, no se han identificado estudios que a partir del análisis del cambio en la respuesta expliquen el significado de los síntomas para los pacientes y su relación con la CdV.

En un estudio previo Serdà (2009) detecta que a pesar del elevado estado comórbido producido por los síntomas, el hombre con cáncer de próstata informaba de una elevada $\mathrm{CdV}^{(8)}$. Esta paradoja podría explicarse a partir de los mecanismos interactivos relativos a la adaptación a la enfermedad. En los últimos años, se han publicado varios estudios que explican los mecanismos que subyacen en el cambio en la respuesta de la $\mathrm{CdV}$ de los enfermos de cáncer ${ }^{(15-17)}$.

Este artículo plantea como objetivo general a) identificar los mecanismos implicados en el cambio en la respuesta de la $\mathrm{CdV}$ en los hombres con cáncer de próstata; y, como objetivo específico b) describir la relación que se establece entre los síntomas y la $\mathrm{CdV}$ de la persona mayor. 


\section{MÉTODO}

\section{Muestra}

La muestra corresponde a un grupo de hombres con cáncer de próstata $(n=66)$. Tras obtener el informe favorable del comité ético de investigación, el programa de ejercicio se realiza en el Hospital de Figueres (España), desde el mes de octubre de 2007 al mes de octubre de 2008.

Para la inclusión de los participantes en el programa es necesario que cumplan dos condiciones:

1. Se requiere el diagnóstico histológico de cáncer de próstata, el estadio de la patología y la fase de tratamiento.

2. Firmar el consentimiento informado del interesado.

Los criterios de exclusión son:

1. Padecer los síntomas previamente al diagnóstico.

2. Presentar déficit cognitivo o trastorno psicótico diagnosticado.

3. La imposibilidad de entender o hablar el español.

La selección es aleatoria entre los participantes que se encuentran en fase de tratamiento y a partir del número de la historia clínica y aplicando el programa SPSS v.15.

\section{Diseño}

La propuesta metodológica se basa en la triangulación entre métodos, por lo que, para el estudio de una misma unidad empírica se combinan dos estrategias de investigación, una cuantitativa y otra cualitativa.

El método cuantitativo se aplica para identificar los cambios en la CdV del hombre con cáncer de próstata, además de estudiar el mecanismo de recalibración implicado en el cambio en la respuesta. El método cualitativo se utiliza para explicar la relación que se establece entre la experiencia vivencial de los síntomas y su impacto con la CdV. Además, describir los mecanismos que explican el cambio en la respuesta en la CdV.

\section{Estudio cuantitativo}

Estudio descriptivo longitudinal. Las muestras de $\mathrm{CdV}$ se recogen en tres series periódicas: P1, P2 y P3.

P1. Pretest. Período correspondiente a la fase de diagnóstico y tratamiento. Se completa el cuestionario sociodemográfico, el de calidad de vida y el de fatiga. Además se incluye la anamnesis, con la objetivación de los síntomas triangulados con la historia clínica.

P2. Posttest. Período que corresponde a la fase de vida autónoma al hogar. Se completan los mismos cuestionarios que en P1 excepto sociodemográfico.

P3. Thentest. Corresponde a la evaluación retrospectiva de P1. Se realiza a las 2 semanas de haber finalizado P2. Este periodo tiene como objetivo que los participantes reevalúen su $\mathrm{CdV}$ en el periodo pretest (P1).

Se completan los mismos cuestionarios que en P2 con las preguntas redactadas con el tiempo verbal en pasado. La aplicación del thentest se realiza para identificar el mecanismo de recalibración en el cambio de la respuesta de la $\mathrm{CdV}^{(16,18)}$. La diferencia de la media entre pretest y thentest proporciona un resultado estimado de la dirección y la magnitud del cambio en la respuesta. Los cambios observados se calculan a partir de la diferencia en la media posttest y pretest mientras que los cambios ajustados se calculan a partir de la media posttest y thentest.

\section{Cuestionarios}

La calidad de vida se evalúa mediante el FACT-P, Functional Assessment Cancer 
Therapy Scale- Prostate [Rango: 0-156] ( $4^{a}$ versión). Este cuestionario incluye 5 dimensiones que corresponden: estado general de salud; ambiente familiar y social; estado emocional; funcionamiento personal; síntomas.

La fatiga se evalúa con la escala FACITescala de cansancio ( $4^{\text {a }}$ versión). [Rango: 0-52].

\section{Evaluación de covariables}

Los síntomas relativos a la enfermedad y el tratamiento se registran con la anamnesis y se contrastan con los registrados en la historia clínica y la observación del procedimiento de respuesta de los participantes realizado por los entrevistadores.

\section{Cálculo del tamaño de la muestra y análisis estadístico}

El tamaño de la muestra calculada para detectar una diferencia entre grupos de 5,0 puntos en el resultado del test FACT-P (desviación estándar DE:9) (19). Aceptando un riesgo alfa de 0,05 y un riesgo beta inferior al 0,05 en un constaste bilateral de medias apareadas, se requieren 53 sujetos para detectar una diferencia igual o superior a 5 unidades. Se asume que la desviación estándar es de 9 . Se ha estimado una tasa de pérdida de seguimiento del $20 \%$.

Para el análisis estadístico de los datos apareados en las variables continuas que siguen una distribución normal, se utiliza el test $t$ de Student. Para el estudio de la correlación entre 2 variables numéricas se ha calculado el índice de correlación de Pearson. Para las variables continuas que no siguen una distribución normal se utiliza el test no paramétrico para datos apareados de Wilcoxon o $U$ de Man-Whitney. Para analizar las variables categóricas se utiliza el test del $X^{2}$. Para evaluar la magnitud de las diferencias entre evaluaciones se aplica el tamaño del efecto d de Cohen, que corresponde a la media de la diferencia entre tests dividido por la desviación típica de la primera evaluación y se interpreta de la forma siguiente: $0,2<\mathrm{d}<0,5$ indica pequeño, $0,5 \leq d<0,8$ moderado y $d \geq 0,8$ un gran tamaño del efecto ${ }^{(20)}$. Los análisis estadísticos se realizan con el programa SPSS versión 15. El nivel de significación escogido es del 5\%.

\section{Estudio cualitativo}

Se realiza el estudio cualitativo siguiendo los principios metodológicos de la Grounded Theory y corresponde a un estudio de $\operatorname{casos}^{(21)}$. Esta propuesta metodológica permite estudiar de una forma retrospectiva el cambio de respuesta

Tabla 1. Casos ejemplares.

\begin{tabular}{|c|c|c|c|c|c|c|c|}
\hline Caso & 1 & 2 & 3 & 4 & 5 & 6 & 7 \\
\hline $\mathrm{n}$ & 9 & 10 & 9 & 10 & 9 & 9 & 10 \\
\hline Edad & $65-70$ & $55-65$ & $55-65$ & $65-70$ & $45-55$ & $75-85$ & $75-85$ \\
\hline Nivel Socio-cultural & No estudios & Superiores & Superiores & Básicos & Superiores & No estudios & Básicos \\
\hline Estado civil & Viudo & Casado & Casado & Casado & Casado & Viudo & Casado \\
\hline Estadio Enfermedad & III-IV & II-III & I-II & I-II & I-II & II-III & III-IV \\
\hline Tipo tratamiento & O & R & P & P & P & BA & BA \\
\hline
\end{tabular}

BA: Bloqueo androgénico; O: Orquiectomía; P: Prostatectomia; R: Radioterapia. 
del cuestionario de $\mathrm{CdV}$, así como, las características de los síntomas desde la percepción del enfermo y su impacto en la CdV.

La muestra se ha construido a partir de un muestro intensivo por representatividad teórica $^{(22)}$. La muestra escogida es de 66 personas con cáncer de próstata distribuida en 7 casos ejemplares que se concretan a partir de la edad, el estadio de la enfermedad, el tipo de tratamiento, el nivel sociocultural, el estado civil. La selección de participantes finaliza al llegar a la saturación de los datos. La distribución final de la muestra por casos corresponde a: caso1 $(n=9)$; caso $2(n=10)$; caso $3(n=9)$; caso 4 $(n=10)$; caso $5(n=9)$; caso $6(n=9)$; caso 7 $(n=10)$. (ver Tabla 1)

La información se ha generado a partir de la entrevista semiestructurada. Las entrevistas se graban magnetofónicamente, con el consentimiento previo de los participantes.

Preguntas: ¿Cómo se encuentra (de salud)?; ¿Su salud se ha visto comprometida con el tratamiento? (Si responde afirmativamente, explique cómo); ¿Cómo describiría su CdV actual?; ¿Cree usted que su CdV ha variado desde el día que le diagnosticaron el cáncer?, en caso afirmativo, ¿Podría explicarme cómo?; ¿Cuál/es es/son la/s esfera/s/dominios o dimensiones más importante/s de su vida?; ¿Cómo ayuda/n o contribuye/n a mantener su CdV?

El procedimiento analítico de la información corresponde a la generación de categorías conceptuales aplicando el Método de Comparaciones Constantes (MCC). Además se realiza la triangulación entre informantes y analistas para incrementar el rigor de los resultados.

\section{RESULTADOS}

\section{Estudio cuantitativo}

La tabla 2, muestra que la edad media de los participantes es de 71,78 años. Presentan un IMC de 28,67 Kg/m². El 93,93\% de los enfermos están diagnosticados entre el estadio II y el estadio III de la enfermedad. La mayoría están casados y jubilados.

Los resultados del cuestionario FACT-P, confirman la disminución significativa de la $\mathrm{CdV}$ entre los periodos p1 (pretest) y p2 (postest). Cuando se procede al análisis de datos en relación a las cinco dimensiones del cuestionario de calidad de vida (FACT-P) y fatiga (FACIT), se observa una disminución significativa en todas las dimensiones de la $\mathrm{CdV}$ entre los periodos P1, P2. (Ver tabla 3)

\section{Análisis de la recalibración de la $\mathrm{CdV}$}

A continuación se presenta el resultado pretest $(\mathrm{P} 1)$ y el resultado thentest (P3) de la $\mathrm{CdV}$ y de cada una de sus dimensiones (ver tabla 4).

La magnitud del cambio detectado entre pretest-thentest es el siguiente: las dimensiones correspondientes al estado emocional, el ambiente familiar y social, la capacidad de funcionamiento personal, el estado general de salud y la calidad de vida se observa una magnitud de cambio pequeña $(0,2<\mathrm{d}<0,5)$; mientras que la magnitud del cambio de la dimensión de los síntomas $(d=0,77)$ es moderada tendiendo a un gran efecto. Finalmente, la magnitud observada en la dimensión de fatiga $(d=0,93)$ es de un gran efecto. (Ver Tabla 5)

Además, se identifica el mecanismo de repriorización en cada una de las dimensiones de la $\mathrm{CdV}$ y periodos evaluados.

En relación a la frecuencia de síntomas y la modalidad de tratamiento, en P1 se observa una media de 4,57 síntomas por participante, con un rango [0-10]. Los 10 síntomas más frecuentes corresponden a: disfunción eréctil (85\%), incontinencia urinaria $(67 \%)$, malestar emocional (58\%), fatiga $(55 \%)$, ginecomastia $(46 \%)$, dolor 
Tabla 2. Características clínicas y epidemiológicas de los participantes.

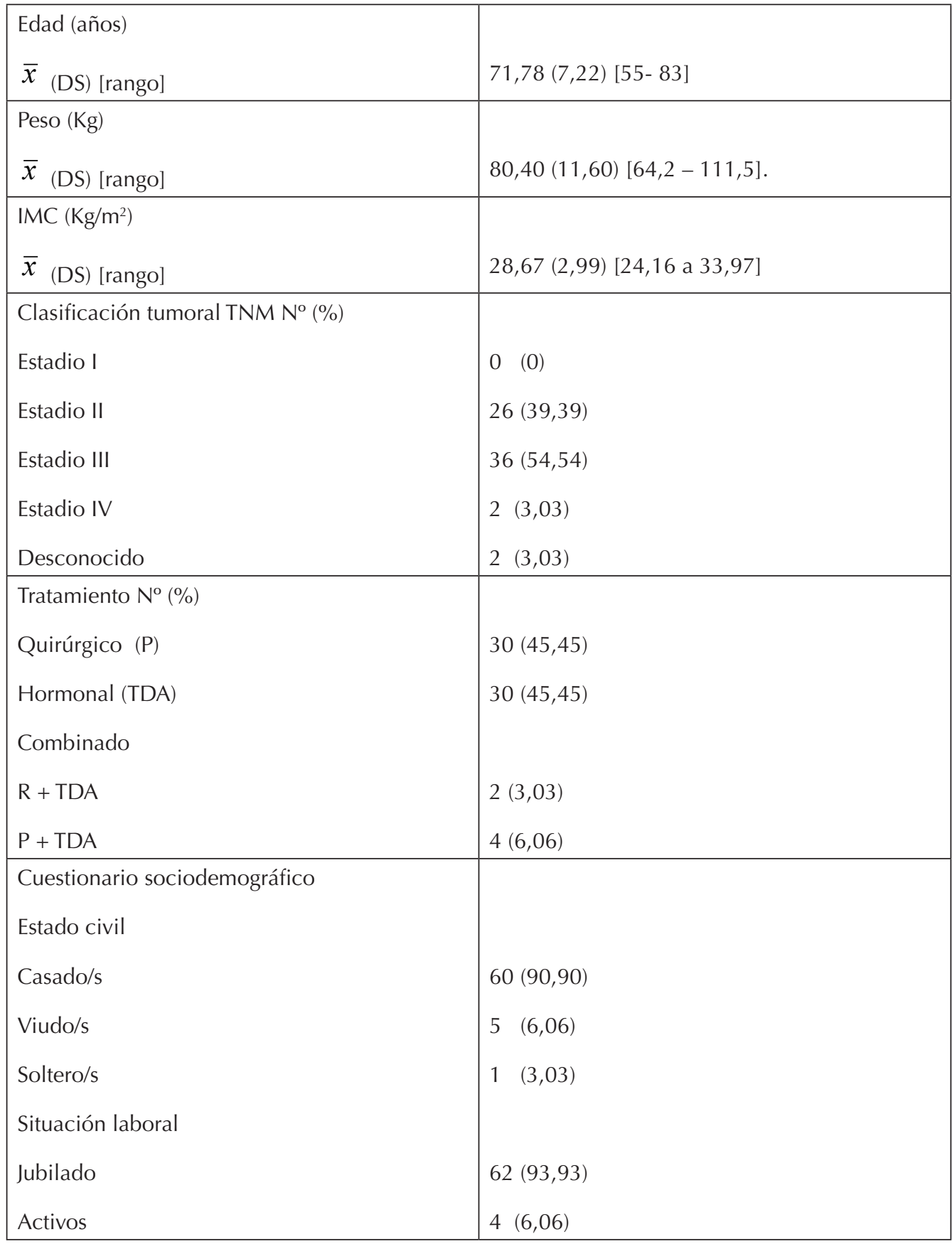

Abreviaturas: IMC, índice de masa corporal; P, prostatectomía; PSA, antígeno prostático específico; P+TDA, prostatectomía con terapia de privación androgénica; $R+T D A$, radioterapia combinada con terapia de privación androgénica; TDA, Terapia de privación androgénica. 


\section{Tabla 3. Cambios en la media y desviación típica (Dt) de las dimensiones de la CdV y la Fatiga.}

\begin{tabular}{|c|c|c|c|c|c|c|c|}
\hline \multicolumn{2}{|c|}{ Descripción Parámetro } & \multicolumn{2}{c|}{ Pretest (p1) } & \multicolumn{2}{c|}{ Postest (p2) } & \multirow{2}{*}{${ }^{*}$} \\
\hline Dimensiones & Rango & $\mathrm{n}$ & $\bar{x}$ & $\mathrm{Dt}$ & $\bar{x}$ & $\mathrm{Dt}$ & $\mathrm{p}^{*}$ \\
\hline FACT-P & {$[0-156]$} & 66 & 108,61 & 18,75 & 101,76 & 19,65 & $<, 001$ \\
\hline Estado Físico General de Salud & {$[0-28]$} & 66 & 23,74 & 3,90 & 21,88 & 4,27 & $<, 001$ \\
\hline Ambiente Familiar y Social & {$[0-28]$} & 66 & 17,95 & 5,41 & 17,33 & 5,58 &, 001 \\
\hline Estado emocional & {$[0-24]$} & 66 & 16,74 & 4,48 & 16,5 & 4,43 & $<, 001$ \\
\hline Capacidad de Funcionamiento Personal & {$[0-28]$} & 66 & 17,39 & 5,17 & 16,32 & 4,8 &, 002 \\
\hline Síntomas & {$[0-48]$} & 66 & 32,77 & 5,77 & 29,73 & 6,88 & $<, 001$ \\
\hline Fatiga & {$[0-52]$} & 66 & 32,64 & 5,66 & 29,05 & 6,85 & $<, 001$ \\
\hline
\end{tabular}

Dt: desviación típica; FACT-P: cuestionario de calidad de Vida del cáncer de próstata; $p^{*}$ : valor de significación $\mathrm{P} \iota, 05 ; \bar{x}$ : media; $\neq$ : cambios observados a las $\mathrm{x}$ semanas del programa de intervención.

Tabla 4. Resultados Pretest-Thentest.

\begin{tabular}{|c|c|c|}
\hline Dimensiones & Pretest & Thentest \\
\hline Calidad de Vida & 108,61 & 100,41 \\
\hline Estado Físico General de Salud & 23,74 & 22,24 \\
\hline Ambiente Familiar y Social & 17,95 & 17,08 \\
\hline Estado emocional & 16,74 & 16,47 \\
\hline $\begin{array}{c}\text { Capacidad de Funcionamiento } \\
\text { Personal }\end{array}$ & 17,39 & 16,26 \\
\hline Síntomas & 32,77 & 28,30 \\
\hline Fatiga & 32,64 & 27,56 \\
\hline
\end{tabular}

Tabla 5. Evaluación del tamaño del efecto d de Cohen.

\begin{tabular}{|c|c|}
\hline Dimensiones & Tamaño del efecto \\
\hline Estado General de Salud & 0,41 \\
\hline Ambiente Familiar y Social & 0,16 \\
\hline Estado emocional & 0,06 \\
\hline Capacidad de Funcionamiento Personal & 0,26 \\
\hline Síntomas & 0,77 \\
\hline Fatiga & 0,93 \\
\hline Calidad de Vida & 0,46 \\
\hline
\end{tabular}




\section{Tabla 6. Resultados del estudio cualitativo.}

P1 $\quad$ En este periodo el objetivo del enfermo es sobrevivir y liberarse del cáncer. El efecto restrictivo determina la trivialización y aceptación de los efectos secundarios retardando su afrontamiento (efecto buffer).

"Al principio sólo piensas en vivir, lo demás lo dejas para luego y piensas ya pasará!".

[Fugas orina]. "Con la sonda no te das cuenta de la gravedad del problema hasta que vuelves a casa".

En este momento el apoyo familiar resulta un factor determinante de la CdV.

"Cuando me sacaron el tumor mi mujer fue mi apoyo. Tenerla cerca, me ayudó a no angustiarme".

P2 Durante este periodo los síntomas generan un mayor impacto en la CdV. Los hombres describen el estado de comórbido a partir de la limitación funcional, incapacidad e interferencia que causan en las actividades habituales de la vida cotidiana.

[Al Ilegar a casa]. "El dolor, la incontinencia, la impotencia me hundieron, no sabía que hacer, estaba agotado. Necesitaba ayuda y no me atreví a pedirla a mis vecinos".

-Los hombres de mayor edad justifican los síntomas utilizando el mecanismo compensatorio de la edad, como por ejemplo con la impotencia sexual. Con la incontinencia describen que es el precio que han de pagar para liberarse de la enfermedad.

"A los 75 años es normal, a quién le importa el sexo en la vida hay otras cosas más importantes, como las caricias o los besos".

El subgrupo de hombres jóvenes manifiesta un mayor distrés secundario a los síntomas. La incontinencia urinaria y la impotencia la identifican como una mutilación. Buscan y elaboran un plan de cuidados integral para rehabilitar los efectos secundarios de la enfermedad.

"Se me escapa la orina. En el hospital no era un gran problema, pero ahora, no puedo hacer nada, es vergonzoso. No tengo sexo, con 50 años, me han desahuciado. Con todo esto cómo se puede vivir! Si lo sé antes, no me opero."

P3 Los hombres identifican una disminución progresiva en la CdV. Este efecto es evidente al reiniciar las actividades de la vida cotidiana (Efecto umbral).

"Cada día iba a peor, resistía menos y tenía más fatiga. Al Ilegar a casa me di cuenta del problema. No podía ni subir las escaleras".

La diferencia de resultados entre P1 y P3 la atribuyen a dos factores. Al tiempo y a la incapacidad que generan los síntomas.

"Con el tiempo te das cuenta que aquello [impotencia] no mejorará. La relación con mi mujer ya no es lo mismo".

El efecto de pérdida no es evidente hasta P3, el periodo clave (umbral) es P2 que actúa como espejo del estado real de salud. El "despertar", al comparar la vida actual con la anterior.

El estado de salud genera respuestas adaptativas y respuestas desadaptativas dependiendo de cada caso en particular. Generalmente las personas jóvenes gestionan un plan de cuidados efectivo, mientras que las personas mayores muestran una mayor resignación.

"He empezado una nueva etapa en mi vida, me pongo pañales para pasear, cuando tengo la fatiga, descanso y no voy a pasear... y así lo llevo día a día"

"A esta edad ya no esperas gran cosa de la vida. 
(40\%), trastorno del sueño $(40 \%)$, pérdida de la libido (40\%), cambios de peso $(16 \%)$, calores y sudores $(16 \%)$.

Al correlacionar el cuestionario FACT-P y el número de síntomas se aplica una correlación bivariada. Los resultados indican que entre la variable FACT-P y el número de síntomas hay una correlación $r=-0,31$, lo que significa que no existe una relación lineal negativa clara entre las dos variables. $p=0,076$

\section{Estudio cualitativo}

En la tabla 6 se muestra los resultados correspondientes a los mecanismos que determinan el cambio en la respuesta de la $\mathrm{CdV}$ considerando los tres periodos estudiados.

\section{DISCUSIÓN}

En virtud de los resultados se confirma la hipótesis que los enfermos con cáncer de próstata presentan una baja $\mathrm{CdV}$ desde el inicio de la enfermedad.

En relación a las dimensiones evaluadas los síntomas y la fatiga son las que se observa una magnitud de disminución mayor.

Así pues, los resultados thentest, confirman una disminución multidimensional en la CdV registrada en el periodo 3 (P3) versus la registrada en el periodo $1(\mathrm{P} 1)^{(23)}$. El mecanismo que determina el cambio en la respuesta de la $\mathrm{CdV}$ corresponde al de recalibración. Este resultado justifica que las personas mayores perciben una baja $\mathrm{CdV}$ desde la fase de diagnóstico de la enfermedad. Este resultado, coincide con otros estudios que evalúan la $\mathrm{CdV}$ en enfermos de cáncer ${ }^{(24)}$. Razmjou (2009) describe que la elevada $\mathrm{CdV}$ al inicio de la enfermedad podría ser debida a la intención de estabilizar el impacto de la curva negativa de la $\mathrm{CdV}^{(25)}$. Este resultado es frecuente entre el subgrupo de perso- nas de mayor edad ${ }^{(14)}$.

Inevitablemente, la disminución global en la $\mathrm{CdV}$ detectada retrospectivamente con el thentest, podría ser debida al sesgo producido al recurrir a la memoria para evaluar un estado anterior ${ }^{(12)}$. Considerando la teoría implícita del cambio, los pacientes no evaluarían la percepción de $\mathrm{CdV}$ en el periodo P1, ya que, el estado de la salud en P2 influiría en el cambio del valor interno final de la respuesta evaluada en P3. Este concepto se enmarca con la teoría del relativismo psicológico ${ }^{(26)}$.

En el periodo 1 (pretest), se registra una $\mathrm{CdV}$ excesivamente elevada considerando la incapacidad que generan los síntomas ${ }^{(8)}$. Los factores que explican este elevado resultado en la $\mathrm{CdV}$ responden a una incapacidad de afrontamiento. En este marco, durante el periodo 1, los afectados describen la CdV como la posibilidad de sobrevivir y liberarse del cáncer $^{(27,28)}$. En este sentido la $\mathrm{CdV}$ se focaliza básicamente a las características y evolución de la enfermedad. Por otro lado, los hombres enfermos sobrevaloran las dimensiones estables correspondientes al estado general de salud, la dimensión social y familiar y la dimensión emocional. El mecanismo implicado en este proceso corresponde al de repriorización. Así pues, inicialmente, el enfermo afronta el diagnóstico y el tratamiento mientras que, los efectos secundarios de la enfermedad se relegan en un segundo plano ${ }^{(14)}$. Sharpe (2005), determina que este estilo de afrontamiento provoca una adaptación parcial e incompleta a la enfermedad ${ }^{(17)}$. El mecanismo psicológico implicado en este proceso se denomina efecto buffer. El sistema sociosanitario sobrevalora el control y estado de la enfermedad en relación a los síntomas. Esta situación imposibilita un control y afrontamiento eficaz de los síntomas. Estos factores provocan que al valorar la CdV durante el P1 el enfermo 
sesgue la dimensión de los síntomas, y no los considere como elemento prioritario en el plan de cuidados de salud. El efecto buffer es limitado y desaparece al diminuir la sensación de riesgo de recidiva y muerte, coincidiendo con el retorno a la vida cotidiana, periodo 2 (P2) ${ }^{(29)}$.

El fracaso en el afrontamiento a la enfermedad es evidente en el periodo 2 (postest), con la disminución significativa multidimensional de la CdV. La magnitud de esta disminución es mayor en los síntomas, fatiga y estado general de salud, mientras que la dimensión emocional y el ambiente familiar y social se mantienen clínicamente estables. Estos resultados coinciden con el estudio de Schwartz et al. ${ }^{(12)}$.

Los hombres justifican el impacto negativo en la dimensión de los síntomas porque en este periodo identifican que los síntomas han adquido una naturaleza crónica, con menos posibilidad de revertir y el impacto en la CdV diaria es mayor. Los resultados confirman que el impacto real de los síntomas en la CdV varía en relación al individuo y la edad.

Así pues, en el subgrupo de hombres jóvenes los síntomas producen un malestar emocional e impacto en la $\mathrm{CdV}$ mayor en relación al subgrupo de hombres de más edad. En este contexto, la disfunción sexual se identifica como una amputación que afecta la relación de pareja. La incontinencia, como una limitación funcional y social que provoca una sensación regresiva, vergüenza y pérdida de autoestima. La fatiga y el dolor, resultan dos síntomas altamente invalidantes e incapacitantes asociados a la recidiva y a la muerte por enfermedad. El malestar emocional que genera este síndrome comporta el inicio de un afrontamiento priorizando los efectos secundarios de la enfermedad $y$, en muchos casos, iniciando un plan de cuidados con intención resolutiva. Este mecanismo corresponde al de repriorización que en este caso tiene un efecto adap- tativo a la enfermedad ${ }^{(30)}$. Esta situación ilustra la visión dinámica y cambiante de la CdV.

En cuanto al subgrupo de mayor edad, la mayoría continúa utilizando el mecanismo de la repriorización de una forma desadaptativa con la intención de estabilizar la CdV. En este contexto, los hombres sobrevaloran las dimensiones menos dañadas, (familia, red social, estado emocional...), mientras que, trivializan los síntomas de la impotencia sexual, la incontinencia urinaria, el dolor y la fatiga sin considerarlos un problema propio de salud $^{(5)}$. Por ejemplo, en el caso de la impotencia se atribuye a un efecto normal natural propio de la edad e identifican las relaciones sexuales como acabadas ${ }^{(31)}$. El impacto del síntoma se resuelve a partir del ajuste marital. En el caso de la incontinencia la naturalizan considerándola como habitual en los hombres de su edad. Determinan que la incontinencia es el efecto secundario residual consecuente de la liberación del tumor al cual hay que resignarse. Los umbrales del dolor y de la fatiga los consideran síntomas cíclicos con los que conviven y resisten. Muchos de ellos conviven con otras enfermedades crónicas lo que justifica el conformismo y la conducta pasiva y resignada que impide el afrontamiento. Esta focalización hacia las dimensiones positivas les ayuda a compensar la pérdida ocasionada por los efectos secundarios de la enfermedad. De acuerdo con Echeteld (2005), la priorización cambiante entre dimensiones de $\mathrm{CdV}$ en el continuo de la enfermedad es un signo claro de deterioro en la $\mathrm{CdV}^{(32)}$. Así pues, el resultado del mecanismo de repriorización en el subgrupo de hombres de mayor edad es en términos generales desadaptativo ${ }^{(17)}$. La aplicación clínica de esta situación consiste en mantener la focalización en las áreas de la vida positivas sin olvidar la planificación de estrategias de intervención oportunas para recuperar 
las áreas más debilitadas.

En este periodo la mayoría de hombres afectados con tumores de bajo riesgo expresan el arrepentimiento de haberse tratado y aseguran que si hubieran podido anticipar las consecuencias no lo hubieran consentido $^{(16)}$.

La dinámica interactiva de los síntomas impide establecer una relación de causaefecto ya que los enfermos describen su actividad de forma global conjunta. Esta relación provoca que la afectación de los síntomas sea borrosa y el impacto que producen en la $\mathrm{CdV}$ varía en cada caso en particular. De acuerdo con Shabbir (2006), consideramos la incontinencia, la impotencia, la fatiga, el dolor y la distimia como un síndrome relativo al cáncer de próstata y el tratamiento que genera un impacto conjunto en la CdV del enfer$\mathrm{mo}^{(33)}$. A este concepto, Molassiotis (2010) lo denomina cluster (efecto en racimo) y el impacto que genera en la CdV es mucho mayor que su acción individual ${ }^{(34)}$. Mostramos desacuerdo con el estudio de Mehnert (2007), el cual consigue establecer una relación lineal entre el número de síntomas y la CdV del enfermo ${ }^{(35)}$.

El mecanismo de reconceptualización coincide cuando se consigue estabilizar la enfermedad. El mecanismo se caracteriza porque el hombre adapta su vida a los efectos de la enfermedad de forma satisfactoria, manifestando el inicio de una nueva etapa post-enfermedad con nuevos propósitos de vida y el describiendo de nuevo el concepto salud y CdV.

A pesar del elevado porcentaje de supervivencia a la enfermedad, este estudio confirma que la $\mathrm{CdV}$ de los pacientes con cáncer de próstata es baja desde la fase de diagnóstico de la enfermedad. El sobrediagnóstico y el sobretratamiento derivado screening conlleva el riesgo adicional que en el continuo de la enfermedad muchos hombres tengan que sufrir unos efectos secundarios y, en consecuencia, una baja $\mathrm{CdV}^{(36)}$.

\section{REFERENCIAS BIBLIOGRÁFICAS}

1. Pla director d'oncologia de Catalunya. Departament de Salut: Direcció General de Planificació i avaluació; 2008.

2. Marcos-Gragera R, Salmeron D, Chirlaque $\mathrm{MD}$, Izarzugaza I, Ardanaz E, Serdà $B$, et al. Tendencia de la supervivencia del cáncer de próstata en España. Gat Sant 2010;(24 (Espec Congr)):116.

3. Welch HG, Albertsen PC. Prostate cancer diagnosis and treatment after the introduction of prostate-specific antigen screening: 1986-2005. J Natl Cancer Inst 2009;101(19):1325-9. Doi: 10.1093/jnci/ djp278

4. Pla director d'oncologia de Catalunya. Departament de Salut: Direcció General de Planificació i avaluació; 2008.

5. Korfage IJ, Hak T, de Koning HJ, EssinkBot ML. Patients' perceptions of the sideeffects of prostate cancer treatment-a qualitative interview study. Soc Sci Med 2006;63(4):911-9. Doi:10.1016/j.socscimed.2006.01.027

6. Ponholzer A, Brossner C, Struhal G, Marszalek M, Madersbacher S. Lower urinary tract symptoms, urinary incontinence, sexual function and quality of life after radical prostatectomy and external beam radiation therapy: real life experience in Austria. World J Urol 2006;24(3):325-30. Doi:10.1007/s00345-006-0075-1

7. Potosky AL, Davis WW, Hoffman RM, Stanford JL, Stephenson RA, Penson DF, et al. Five-year outcomes after prostatectomy or radiotherapy for prostate cancer: the prostate cancer outcomes study. J Natl Cancer Inst 2004;96(18):1358-67. Doi:10.1093/jnci/djh259

8. Serdà FB. Avaluació dels efectes d'un programa d'exercici físic individualitzat sobre la Qualitat de Vida (QdV), la incontinència urinària, la fatiga $i$ la resistència muscular amb malalts de càncer de pròstata. TESEO: Universitat de 
Girona (UdG); 2009.

9. Cella DF, Tulsky DS. Quality of life in cancer: definition, purpose, and method of measurement. Cancer Invest 1993;11(3):327-36.

10. Díaz Veiga P, Facal J, Yanguas Leazón JJ. Funcionamiento psicológico y envejecimiento. Aprendizajes a partir de estudios longitudinales. Rev. Esp Geriatr Gerontol 2010;45(6):350-7. Doi:10.1016/j. regg.2010.09.001

11. Literas L., Navarro A, Fontanals MD. Diseño y validación de una escala de satisfacción y calidad de vida para usuarios de centros residenciales y sociosanitarios. Rev. Esp Geriatr Gerontol 2010;45(6):32035. Doi:10.1016/j.regg.2010.04.008

12. Schwartz CE, Bode R, Repucci N, Becker J, Sprangers MA, Fayers PM. The clinical significance of adaptation to changing health: A meta-analysis of response shift. Qual Life Res 2006;15(9):1533-50.

13. Sprangers MA, Schwartz CE. Integrating response shift into health-related quality of life research: A theoretical model. Soc Sci Med 1999;48(11):1507-15.

14. Kvam AK, Wisloff F, Fayers PM. Minimal important differences and response shift in health-related quality of life; a longitudinal study in patients with multiple myeloma. Health Qual Life Outcomes 2010;8:79.

15. Hagedoorn M, Sneeuw KC, Aaronson NK. Changes in physical functioning and quality of life in patients with cancer: Response shift and relative evaluation of one's condition. J Clin Epidemiol 2002;55(2):176-83.

16. Korfage IJ, de Koning HJ, Essink-Bot ML. Response shift due to diagnosis and primary treatment of localized prostate cancer: A then-test and a vignette study. Qual Life Res 2007;16(10):1627-34. Doi: 10.1007/ s11136-007-9265-6

17. Sharpe L, Butow P, Smith C, McConnell D, Clarke S. Changes in quality of life in patients with advanced cancer: Evidence of response shift and response restriction. J Psychosom Res 2005;58(6):497-504.
Doi.1016/j.jpsychores.2005.02.017

18. Schwartz CE, Sprangers MA. Guidelines for improving the stringency of response shift research using the thentest. Qual Life Res 2010;19(4):455-64.

19. Segal RJ, Reid RD, Courneya KS, Malone SC, Parliament MB, Scott CG, et al. Resistance exercise in men receiving androgen deprivation therapy for prostate cancer. J Clin Oncol 2003;21(9):1653-9.

20. Cohen J. Statistical power analysis for the behavioral sciences. Hillsdale ed. NJ: Erlbaum: 1988.

21. Denzin N, Lincoln Y. Handbook of qualitative research (2nd ed.). Thousand Oaks ed. CA: 2000.

22. Taylor SJ, Bogdan R. A qualitative approach to the study of community adjustment. Monogr Am Assoc Ment Defic 1981;(4):71-81.

23. Calman KC. Quality of life in cancer patients--an hypothesis. J Med Ethics 1984;10(3):124-7.

24. Echteld MA, Deliens L, Ooms ME, Ribbe MW, van der WG. Quality of life change and response shift in patients admitted to palliative care units: a pilot study. Palliat Med 2005;19(5):381-8.

25. Razmjou H, Schwartz CE, Yee A, Finkelstein JA. Traditional assessment of health outcome following total knee arthroplasty was confounded by response shift phenomenon. J Clin Epidemiol 2009;62(1):916. Doi:10.1016/j.jclinepi.2008.08.004

26. Norman G. Hi! How are you? Response shift, implicit theories and differing epistemologies. Qual Life Res 2003;12(3):23949.

27. Chen RC, Clark JA, Talcott JA. Individualizing quality-of-life outcomes reporting: How localized prostate cancer treatments affect patients with different levels of baseline urinary, bowel, and sexual function. J Clin Oncol 2009;27(24):3916-22.

28. Talcott JA. Prostate cancer quality of life: Beyond initial treatment and the patient. J Clin Oncol 2007;25(27):4155-6. 
29. Lepore SJ, Helgeson VS, Eton DT, Schulz R. Improving quality of life in men with prostate cancer: a randomized controlled trial of group education interventions. Health Psychol 2003;22(5):443-52. Doi:10.1037/0278-6133.22.5.443

30. Cowen ME, Miles BJ, Cahill DF, Giesler RB, Beck JR, Kattan MW. The danger of applying group-level utilities in decision analyses of the treatment of localized prostate cancer in individual patients. Med Decis Making 1998;18(4):376-80. Doi:10.1177/0272989X9801800404

31. Korfage IJ, Roobol M, de Koning HJ, Kirkels WJ, Schroder FH, Essink-Bot ML. Does "normal" aging imply urinary, bowel, and erectile dysfunction? A general population survey. Urology 2008;72(1):3-9. Doi: 10.1016/j.urology.2008.01.058,

32. Echteld MA, van ZL, Bannink M, Witkamp E, van der Rijt CC. Changes in and correlates of individual quality of life in advanced cancer patients admitted to an academic unit for palliative care. Palliat Med 2007;21(3):199-205.
Doi:10.1177/1352458507077404

33. Shabbir MA, Sven Gogov, Zishan Allibhai. Long-term side effects of androgen deprivation therapy in men with non-metastatic prostate cancer: A systematic literature review. Crit Rev Oncog 2006;60:201-15. Doi: 10.1016/j.critrevonc.2006.06.006

34. Molassiotis A, Wengstrom $\mathrm{Y}$, Kearney $\mathrm{N}$. Symptom cluster patterns during the first year after diagnosis with cancer. J Pain Symptom Manage 2010;39(5):847-58. doi:10.1016/j.jpainsymman.2009.09.012

35. Mehnert A, Lehmann C, Schulte T, Koch $U$. Presence of symptom distress and prostate cancer-related anxiety in patients at the beginning of cancer rehabilitation. Onkologie 2007;30(11):551-6. Doi:10.1159/000108578

36. Draisma G, Boer R, Otto SJ, van dC, I, Damhuis RA, Schroder FH, et al. Lead times and overdetection due to prostatespecific antigen screening: estimates from the European Randomized Study of Screening for Prostate Cancer. J Natl Cancer Inst 2003;95(12):868-78. Doi: 10.1093/jnci/95.12.868 
